\title{
nature neurosciencegateway
}

\section{FEATURED ARTICLES}

Smells like love

Neuroscience Gateway (August 2007) | doi:10.1038/aba1769

Female mice with impaired pheromone sensation show maletypical sexual behaviors.

In Scent of a Woman, Al Pacino plays a grouchy blind man captivated by a woman's perfume. In animals, pheromone sensation is important for aggressive and mating behaviors. Now Kimchi et al. report that female mice with deficits in pheromone perception show male-typical sexual behavior toward both male and female mice in a recent article in Nature.

In the development of neural circuits responsible for sex behavior, the female circuit is

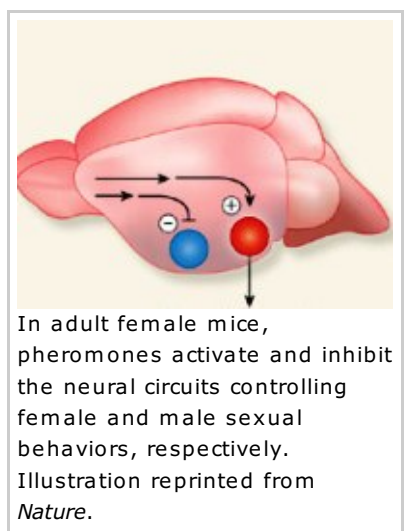
considered the default. Exposure to testosterone and its metabolites during a limited developmental window permanently masculinizes these circuits. Re-exposure to testosterone in the adult activates male sex behaviors. Exogenous testosterone does not induce male behaviors without developmental exposure.

Pheromones act in the main olfactory epithelium and the vomeronasal organ (VNO). VNO neurons specifically express the calcium channel TRPC2. Male mice lacking TRPC2 court both female and male mice and show reduced aggressive behavior in response to male intruders relative to wild-type males, suggesting that the VNO is important in aggression and the selection of mating partners of the opposite sex.

What about females? The authors found that female Trpc2 knockout mice showed male-specific sex behavior toward both male and female mice, behaving identically to male $\operatorname{Trpc} 2$ knockout mice. Male mounting behavior can be a sign of dominance. However, female Trpc2 knockout mice also showed male-typical vocalization, sniffing and motor behaviors, which are rarely associated with activities other than mating. Together, these data suggest that $\operatorname{Trpc} 2$ acts during development to organize male sex behavior. However, surgical removal of the VNO in adult females produced sex behavior similar to that of female Trpc2 knockout mice, suggesting that the neural circuits that mediate male sex behavior persist in the adult brains of female mice.

When housed in groups, Trpc2 knockout and heterozygous female mice did not develop a social hierarchy, suggesting that the maletypical sex behavior in female Trpc2 knockout mice is not related to dominance. When male intruders entered the group environment, both Trpc2 knockout and heterozygous females showed aggressive behaviors. However, only Trpc2 knockout females chased, sniffed and mounted the male intruders. Most females, regardless of genotype, became pregnant after males entered the arena, suggesting that female sex behavior persists in female Trpc2 knockout mice. 
TRPC2 is also important in maternal behaviors. All lactating Trpc2 heterozygous mice attacked male intruders. In contrast, lactating Trpc2 knockout mice were sexually receptive toward male

intruders. Trpc2 knockout females spent less time with their pups than did Trpc2 heterozygotes, suggesting that pheromone perception is important in maternal behaviors.

Relative to female $\operatorname{Trpc} 2$ heterozygous mice, female Trpc2 knockout mice showed slight increases in free testosterone. However, female Trpc2 knockout mice had far less testosterone than do normal males or than is necessary to activate male behaviors in masculinized female mice.

Therefore, these data indicate that the neural circuits mediating male behaviors are latent in the adult female brain. The authors suggest that latent neural circuits that control female behaviors might also be found in brains of male mice.

Debra Speert

1. Kimchi, T., Xu, J. \& Dulac, C. A functional circuit underlying male sexual behaviour in the female mouse brain. Nature doi: 10.1038/nature06089 (2007). | Article | 